\title{
Quenched Phonon Drag in Silicon Nanowires Reveals Significant Effect in the Bulk at Room Temperature
}

\author{
Jyothi Sadhu, ${ }^{\dagger}$ Hongxiang Tian, ${ }^{\dagger}$ Jun Ma, $^{\dagger}$ Bruno Azeredo, $^{\dagger}$ Junhwan Kim, $^{\dagger}$ Karthik Balasundaram, ${ }^{\ddagger}$ \\ Chen Zhang, ${ }^{\ddagger}$ Xiuling Li, ${ }^{\ddagger}$, P. M. Ferreira, ${ }^{\dagger}$ and S. Sinha ${ }^{*},, \S$
}

${ }^{\dagger}$ Department of Mechanical Science and Engineering, ${ }^{\ddagger}$ Department of Electrical and Computer Engineering, and ${ }^{\S} \mathrm{Micro}$ and Nanotechnology Laboratory, University of Ilinois at Urbana-Champaign, Urbana Illinois 61801, United States

Supporting Information
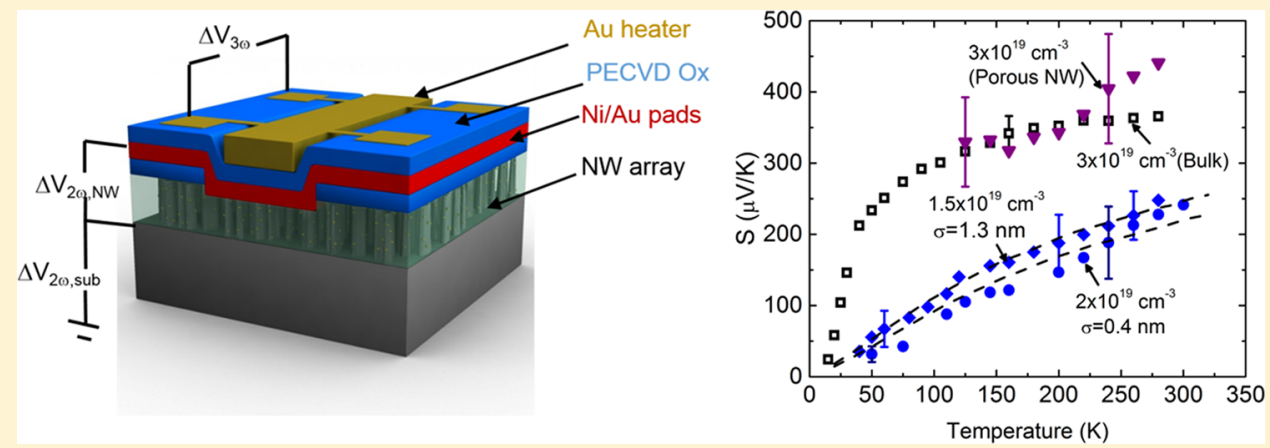

ABSTRACT: Existing theory and data cannot quantify the contribution of phonon drag to the Seebeck coefficient $(S)$ in semiconductors at room temperature. We show that this is possible through comparative measurements between nanowires and the bulk. Phonon boundary scattering completely quenches phonon drag in silicon nanowires enabling quantification of its contribution to $\mathrm{S}$ in bulk silicon in the range $25-500 \mathrm{~K}$. The contribution is surprisingly large $(\sim 34 \%)$ at $300 \mathrm{~K}$ even at doping of $\sim 3 \times 10^{19} \mathrm{~cm}^{-3}$. Our results contradict the notion that phonon drag is negligible in degenerate semiconductors at temperatures relevant for thermoelectric energy conversion. A revised theory of electron-phonon momentum exchange that accounts for a phonon mean free path spectrum agrees well with the data.

KEYWORDS: Seebeck effect, phonon drag, silicon nanowires, thermoelectrics, electron-phonon scattering

uantifying the Seebeck coefficient, $S$, in semiconductors across a broad temperature range poses a rigorous test of fundamental nonequilibrium theory and is important in engineering thermoelectric ${ }^{1-4}$ and semiconductor devices. ${ }^{5}$ The Seebeck effect arising from the diffusion of charge carriers along a temperature gradient is enhanced by the drag imposed on carriers from the accompanying diffusion of phonons. Reflecting this, $S$ is often expressed as a sum of two contributions, ${ }^{6}$ charge diffusion $\left(S_{\mathrm{d}}\right)$ and phonon drag $\left(S_{\mathrm{ph}}\right)$. Quantifying $S_{\mathrm{ph}}$ in bulk semiconductors has not received much attention beyond the initial work, ${ }^{6-9}$ possibly due to a narrative that $S_{\mathrm{ph}} \ll S_{\mathrm{d}}$ at the high doping levels ${ }^{10-12}$ and temperatures required for practical thermoelectric energy conversion. In the first derivation provided by Herring, ${ }^{6} S_{\mathrm{ph}}=\beta u_{0} \bar{\Lambda} / \mu_{\mathrm{e}} T$ where $\bar{\Lambda}$ is mean free path (MFP) of phonons participating in drag, $\mu_{\mathrm{e}}$ is the electron mobility, $u_{0}$ is speed of sound, and $\beta$ is the relative strength of electron-phonon scattering with respect to the ionic impurity scattering of electrons. Herring's formula leads to the interpretation that $S_{\mathrm{ph}} \sim 0$ in degenerate semiconductors when (i) assuming $\beta \ll 1$, based on the Conwell-Weisskopf (CW) limit ${ }^{13}$ of ionic impurity scattering; (ii) underestimating the MFP of long wavelength phonons and; (iii) not accounting for a phonon MFP spectrum $\Lambda(\omega)$ of drag phonons. Contrary to the theory, the first experimental data ${ }^{7}$ in near-degenerate $\mathrm{Si}$ $\left(\sim 10^{18} \mathrm{~cm}^{-3}\right)$ shows $S_{\mathrm{ph}}$ contributes $\sim 50 \%$ of $S$ at $300 \mathrm{~K}$. The quantitative understanding of phonon drag remains elusive even 60 years after the initial exposition, both in bulk semiconductors and more importantly at the nanoscale. With the clearer picture of $\Lambda(\omega)$ starting to emerge for several materials, ${ }^{14,15}$ reexamining phonon drag and its magnitude is of immediate physical and technological interest.

However, direct measurements of $S_{\mathrm{ph}}$ are unknown even for bulk semiconductors. Existing experiments derive $S_{\text {ph }}$ indirectly by subtracting a theoretical $S_{\mathrm{d}}$ from the measured $S$. The separation of $S$ into $S_{\mathrm{d}}+S_{\mathrm{ph}}$ implicitly assumes phonons reach stationary equilibrium by Umklapp processes more rapidly than electrons, a condition only true in nondegenerate semiconductors. At higher carrier concentrations $\left(>10^{18} \mathrm{~cm}^{-3}\right.$ for $\mathrm{Si}$ at $300 \mathrm{~K})$ though, $^{16}$ simultaneous electron-phonon nonequilibrium can erase any unique distinction between $S_{\mathrm{d}}$ and $S_{\mathrm{ph}}$. Consideration of simultaneous nonequilibrium is then necessary to rigorously satisfy the Kelvin-Onsager relations. ${ }^{17}$

Received: January 22, 2015

Revised: March 22, 2015

Published: April 1, 2015 
This complicates an unambiguous extraction of $S_{\mathrm{ph}}$ from $S$ data. In this Letter, we show that comparative measurements of $S$ in the bulk and in nanowires resolve this issue even in highly doped Si. In nanowires, phonon boundary scattering drives the faster equilibration of phonons, decoupling $S$ into the simple sum $S_{\mathrm{d}}+S_{\mathrm{ph}}$, and also progressively reduces $S_{\mathrm{ph}}$ with decreasing dimensions. In narrow wires $(\lesssim 100 \mathrm{~nm}), S_{\mathrm{ph}}$ is completely quenched across a wide temperature range $(\sim 25-500 \mathrm{~K})$. This shows the comparison of measurements on such nanowires against those on equivalently doped bulk yields $S_{\mathrm{ph}}$ in the bulk. Contrary to the common narrative, we find that $S_{\mathrm{ph}}$ contributes significantly to $S$ in bulk $\mathrm{Si}(\sim 34 \%$ of $S)$ even at $300 \mathrm{~K}$ and $\sim 3$ $\times 10^{19} \mathrm{~cm}^{-3}$ doping. Quantitative understanding of $S_{\mathrm{ph}}$ in the bulk is a major step toward understanding drag in lowdimensional materials and nanostructures.

The fundamental insight guiding our experiments is that there exists a crystal size below which $S_{\text {ph }} \sim 0$ across a wide range of temperatures. We obtain approximate quantitative estimate of this size from Herring's classic theory ${ }^{6}$ Herring models drag in Peltier framework ( -approach, a reserve of Seebeck effect) where phonon drag is the thermal flux set up in an isothermal conductor by an unit electric current. The thermal flux is a result of a balance of the momentum added to phonons through electron-phonon processes and that destroyed through phonon-phonon processes. $S_{\mathrm{ph}}$ is proportional to the spectral average of the net change in phonon momentum $\Delta P_{0}(q)$ and depends on the phonon MFP spectrum $\tau(q)$ where $q$ is phonon wavenumber. While the specific form of $\Delta P_{0}(q)$ is complicated, its scaling with the boundary length, $l_{\mathrm{b}}$ is readily obtained. Assuming phonons are propagating in a cylinder wire (along $x$-axis) of radius $l_{\mathrm{b}}$, the net crystal momentum change $\Delta P(q ; r)$ due to boundary scattering is related to that in bulk $\Delta P_{0}(q)$ by the Boltzmann equation for momentum transfer ${ }^{6}$

$$
v_{y} \frac{\partial \Delta P}{\partial y}+v_{z} \frac{\partial \Delta P}{\partial z}=-\frac{\Delta P-\Delta P_{0}}{\tau(q)}
$$

where $\tau_{\mathrm{q}}^{-1}$ is the phonon scattering rate of mode $q$ in absence of boundary scattering. We solve for $\Delta P$ in eq 1 assuming diffuse boundaries such that $\Delta P\left(q ; l_{\mathrm{b}}\right)=0$ (see Supporting Information $S 1)$. The spectral and radial average of net momentum change of each mode, $\Delta \bar{P}(q ; r)$ is proportional to $S_{\mathrm{ph}}$ in nanowire. We define a scattering ratio $\gamma=\Delta \bar{P} / \Delta \bar{P}_{0}$ that quantifies the scaling of $S_{\text {ph }}$ with boundary scattering. Figure 1 plots $\gamma$ with crystal size $l_{\mathrm{b}}$ at $300 \mathrm{~K}$, showing that the boundary scattering starts

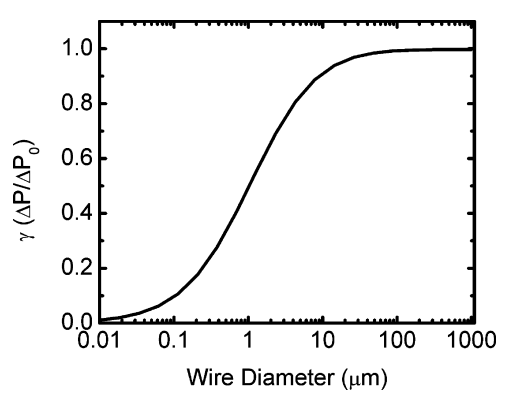

Figure 1. Phonon drag quenching by boundary scattering represented by $\gamma$, the ratio of total crystal momentum $\Delta \bar{P}$ in low-dimensional wires in comparison to that in bulk $\Delta \bar{P}_{0} . S_{\text {ph }}$ reduces by boundary scattering at dimensions below $\sim 10 \mu \mathrm{m}$ at $300 \mathrm{~K}$. Validated Umklapp scattering rates are considered for $\tau_{\mathrm{q}}^{-1}$ with $\tau_{\text {umk }}^{-1}=1.6 \times 10^{-17} \omega^{2}$ for longitudinal and $2.5 \times 10^{-17} \omega^{2}$ for transverse modes at $300 \mathrm{~K}^{39}$ affecting $S_{\text {ph }}$ at wire radii $<10 \mu \mathrm{m}$. Drag is completely quenched as the wire radius approaches $\sim 100 \mathrm{~nm}$. In this Letter, we show that indeed this is the case and by comparing $S$ in $\sim 100 \mathrm{~nm}$ diameter wire with that in the bulk at the same doping, we directly quantify $S_{\mathrm{ph}}$ in the bulk.

We employed a frequency-domain technique ${ }^{18,19}$ to perform cross-plane measurements of $S$ in silicon nanowire ( $\mathrm{SiNW}$ ) arrays. This is in contrast to steady state, in-plane measurements of $S$ in individual or few nanowires in previous work, ${ }^{20,21}$ where both electrical and thermal contact resistances can be relatively large and introduce significant errors, often difficult to quantify. The cross-plane geometry enables measurements from a top contact of $\sim \mathrm{mm}^{2}$ area over the nanowire array. Cross plane measurements by the well-known Harman technique $e^{22,23}$ suffer from parasitic joule heating at the contacts and nonuniform current injection through the sample complicates the extraction of $S$. We avoid these issues by employing instead an open-circuit measurement based on frequency dependent heating. Steady periodic heating creates a frequency dependent temperature distribution across a SiNW array fabricated on top of a Si substrate. The open circuit Seebeck voltages developed across the array, the substrate and any contacts have distinguishable frequency responses due to variable heat penetration in each layer. The geometry further facilitated secondary ion mass spectrometry (SIMS) for measuring dopant concentrations, important in estimating $S_{\mathrm{d}}$. In past work, ${ }^{20}$ carrier concentration has been inferred from resistivity measurements but known issues complicate a reliable extraction. $^{24}$

Metal-assisted chemical etching on low-doped Si provided silicon nanowire arrays (SiNWs) with mean diameter $120 \mathrm{~nm}$ and standard deviation $20 \mathrm{~nm} .^{25}$ The arrays were subsequently doped ex situ ${ }^{26}$ and the dopant concentrations in the SiNW arrays was characterized using secondary ion mass spectroscopy (see Supporting Information S2). Figure 2a shows the schematic of the measurement platform. Joule heating in the line heater using an ac current $(\sim 40 \mathrm{~mA})$ at frequency $\omega$ set up temperature oscillations across the sample and the heater $\left(\Delta T_{\mathrm{h}}\right)$ at frequency $2 \omega$. The Seebeck voltage across the SiNW array $\left(\Delta V_{\mathrm{NW}}\right)$ and the substrate $\left(\Delta V_{\text {sub }}\right)$ also oscillate at $2 \omega$ and is measured by a lock-in amplifier to yield an open-circuit voltage, $V_{2 \omega}=\Delta V_{\mathrm{NW}}+\Delta V_{\text {sub }}$. In order to isolate the contribution of the SiNW array from that of the substrate, we measured an additional reference sample comprising only the substrate with the same insulation layer. Comparing the reference and the NW array sample, we could differentially extract the temperature drop across the SiNW array $\left(\Delta T_{\mathrm{NW}}=\right.$ $\left.\Delta T_{\mathrm{h}, \mathrm{NW}}-\Delta T_{\mathrm{h}, \mathrm{ref}}\right)$ and its Seebeck voltage contribution $\left(\Delta V_{\mathrm{NW}}\right.$ $\left.=V_{2 \omega, \mathrm{NW}}-V_{2 \omega, \mathrm{ref}}\right)$ to obtain the Seebeck coefficient of the array $\left(S_{\mathrm{NW}}=\Delta V_{\mathrm{NW}} / \Delta T_{\mathrm{NW}}\right)$. Good fits to the data of $\Delta T_{\mathrm{h}}$ and $V_{2 \omega}$ with modulation frequency using standard $3 \omega$ heat diffusion models show negligible contributions from the contacts in our measurements on bulk and NWA samples (see Supporting Information S3). The thermal conductivity of the SOG filled array is simultaneously obtained as $k_{\text {ARRAY }}=$ $P h_{\mathrm{NW}} /\left(2 b L_{\mathrm{h}} \Delta T_{\mathrm{NW}}\right)$ where $h_{\mathrm{NW}}$ is the height of NW array, $L_{\mathrm{h}}$ and $2 b$ are the length and the full width of the heater line with heating power $P$. The thermal conductivity of NW array is calculated from the areal coverage $(x)$ of NWs as $k_{\mathrm{ARRAY}}=x k_{\mathrm{NW}}$ $+(1-x) k_{\mathrm{SOG}}$. The differential measurement scheme requires the thermal penetration depth to be smaller than the substrate thickness and the length of the heater in both the reference and the SiNW samples. Selecting appropriate frequency windows at 


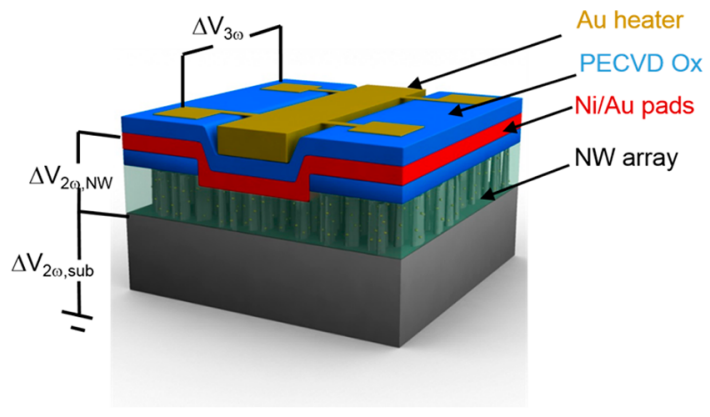

(a)

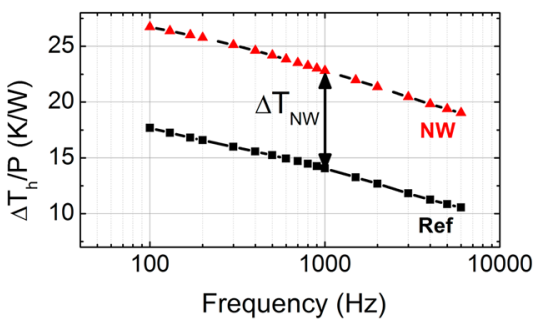

(b)

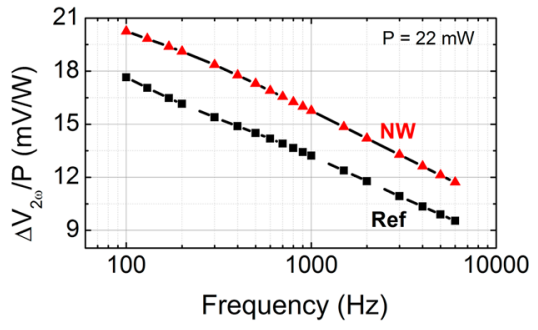

(c)

Figure 2. (a) Schematic showing the platform for the simultaneous measurement of Seebeck coefficient and thermal conductivity on the spin-on glass filled nanowires. The frequency response of the temperature rise of the heater (b) and the measured Seebeck voltage (c) normalized by heating power at $300 \mathrm{~K}$

different temperatures ensures this condition. Figure $2 b, c$ illustrates the frequency responses of the heater temperature rise and the Seebeck voltage respectively in measurements conducted at $300 \mathrm{~K}$. Measurements at $300 \mathrm{~K}$ employed frequencies in the range $200-4000 \mathrm{~Hz}$ but those at low temperature, for example, $80 \mathrm{~K}$, required a frequency window $>1 \mathrm{kHz}$.

Figure 3a shows the temperature trend of the measured $S$ for bulk $\mathrm{Si}$ and $p$-type NW arrays doped to $\sim 10^{19} \mathrm{~cm}^{-3}$ with surface roughness height of $0.4 \mathrm{~nm}$ (diameter $d=108 \mathrm{~nm}$ ) and $1.3 \mathrm{~nm}(d=98 \mathrm{~nm})$ respectively. We find that $S$ in SiNWs decreases by $\sim 62 \%$ at $100 \mathrm{~K}$ and by $\sim 34 \%$ at $300 \mathrm{~K}$ irrespective of roughness. The temperature trend of $S$ is markedly different than the trend in the bulk and, as discussed later, is consistent with the argument that phonon drag is quenched in nanowires. Figure 3a also shows $S$ measured on mesoporous nanowires obtained through direct metal-assisted etching of a degenerate Si wafer ${ }^{27}\left(N=3 \times 10^{19} \mathrm{~cm}^{-3}\right)$. Interestingly, $S$ at $300 \mathrm{~K}$ in this case is similar in magnitude to that in the bulk $\mathrm{Si}$ but possesses a steeper slope with respect to temperature. We varied doping in SiNWs in the degenerate range to further investigate the trends. Figure $3 \mathrm{~b}$ shows the Seebeck measurements in the temperature range $300-450 \mathrm{~K}$ on smooth nanowire arrays $(d=$ $100-125 \mathrm{~nm}$ ) doped between $3 \times 10^{18}$ and $7 \times 10^{19} \mathrm{~cm}^{-3}$. Table 1 summarizes the details of the samples used in this work.

To understand the reduction in $S$ in SiNW relative to the bulk across all doping, we first calculate the diffusion component, $S_{\mathrm{d}}$, in the wires. The diffusion component is the average energy transported by the carriers relative to the Fermi level $\left(E_{\mathrm{F}}\right)$ and assuming phonon equilibrium ${ }^{28}$

$$
e S_{\mathrm{d}} T=\frac{\int_{0}^{\infty} \mathrm{d} E f_{0}^{\prime} \tau_{\mathrm{e}} v_{x}^{2} D(E)\left(E-E_{\mathrm{F}}\right)}{\int_{0}^{\infty} \mathrm{d} E f_{0}^{\prime} \tau_{\mathrm{e}} v_{x}^{2} D(E)}=\left\langle E-E_{\mathrm{F}}\right\rangle
$$

where $D(E)$ is three-dimensional (3D) density of states, $f_{0}^{\prime}$ is the energy derivative of the Fermi distribution $f_{0}, E_{\mathrm{F}}$ is the Fermi level relative to band edge, $v_{x}$ is electron velocity, $\langle *\rangle$ is the average over the electron relaxation time $\tau(\mathrm{E})$, which is obtained from the literature ${ }^{29,30}$ (see Supporting Information S4). The dashed lines in Figure 3a show the $S_{\mathrm{d}}$ calculated using eq 2. The temperature-dependent electron scattering rate $\tau^{-1}(E)$ is obtained using Mattheissen's sum of electron (hole)phonon, ionic impurity, intervalley, plasma, and boundary scattering rates. ${ }^{31}$ When considering scattering from ionic impurities, a simple revision to the Brooks-Herring formula ${ }^{32}$ qualitatively corrects the erroneous narrative of $\beta \ll 1$ obtained from the CW limit at high doping. For solid NWs in Figure 3a, we found the SiNW data to be in good agreement with $S_{\mathrm{d}}$ calculated at the same doping concentration as measured from SIMS. The carrier concentration was also verified using single nanowire measurements where possible (see Supporting Information S5). For calculating $S_{\mathrm{d}}$ at higher temperatures $(300-450 \mathrm{~K})$, it is useful to express $\tau_{\mathrm{e}} \propto E^{r-1 / 2}$ or (electron $\operatorname{MFP} \Lambda_{\mathrm{e}} \sim E^{\mathrm{r}}$ ) where the "scattering exponent" $r$ can vary from 0 to 2 depending on the dominant scattering process. The dashed lines in Figure $3 \mathrm{~b}$ shows $S_{\mathrm{d}}$ calculated from eq 2 by fitting the parameter $r$ in $\tau(E)$. We find that the best fit value of $r$ varies between 0.7 at low doping $\left(N=3 \times 10^{18} \mathrm{~cm}^{-3}\right)$ to 1.2 at high doping $\left(N=7 \times 10^{19} \mathrm{~cm}^{-3}\right)$. The increase in $r$ with doping reflects the increasing dominance of ionic impurity scattering over longitudinal acoustic phonon and boundary scattering.

Previous reports on Seebeck effect in nanowires suggested ${ }^{21}$ that nanowires can exhibit an anomalous phonon drag enhancement. The present work contradicts this possibility; instead, boundary scattering reduces phonon drag as expected. In contrary, we observe larger magnitude and steeper trend of $S$ in wires obtained by directly etching highly doped $\mathrm{Si}$, similar to data reported by Hochbaum et.al. ${ }^{20}$ Such steep $S$ versus $T$ trend is unphysical for uncompensated $c$-Si and we attribute the result to porosity in the wires. Similarly, we also find the thermal 


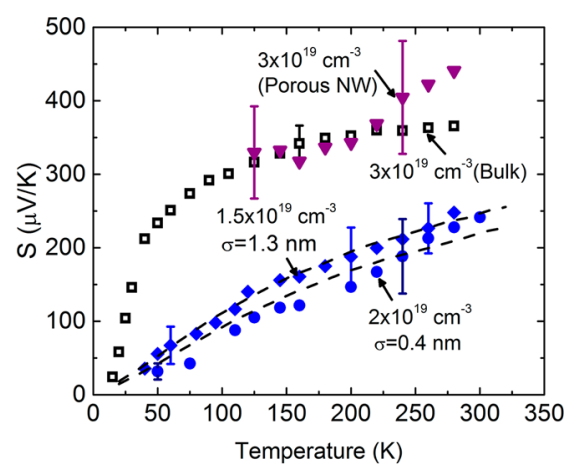

(a)

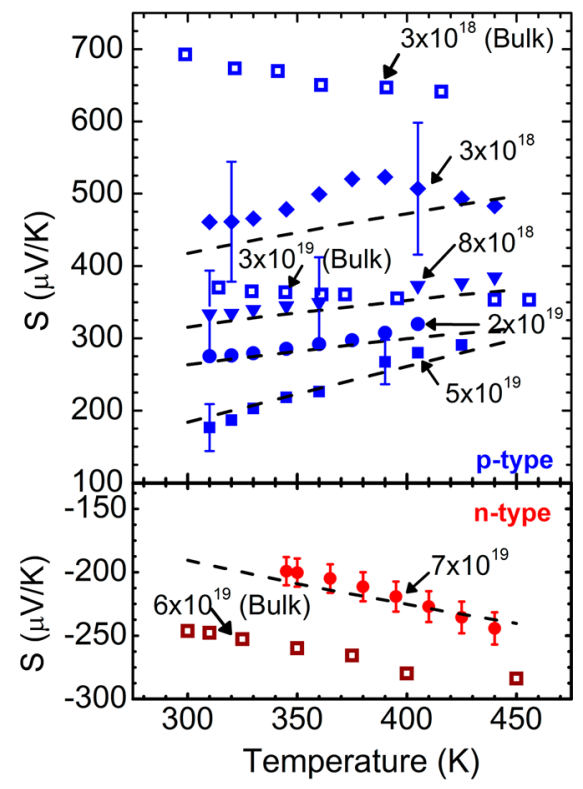

(b)

Figure 3. Seebeck coefficient of NW arrays (a) temperature dependent and (b) measured across several doping concentrations. The dashed lines represent the diffusion component, $S_{\mathrm{d}}$ calculated theoretically using eq 2 . The open symbols represent data for bulk Si.

conductivity $\left(k_{\mathrm{NW}}\right)$ of these wires dramatically reduced to 2.6 $\mathrm{W} / \mathrm{mK}$ (Table 1) similar to the ref 20 while the solid NWs have $k_{\mathrm{NW}}$ close to Casimir limit for doped wires. To understand the anomalous trend of $S$ in the mesoporous wires, we measured the electrical resistivity of the NW arrays versus temperature (see Supporting Information Figure S5). The temperature trend of resistivity and $S_{\mathrm{d}}$ are sensitive to the energy exponent of the electron scattering rate while their magnitudes depend on Fermi level. Assuming a fixed carrier concentration, we fit the resistivity using energy exponent $r$ and obtained the best fit value as $r=2.2$, consistent with previously reported carrier scattering in bulk porous silicon. ${ }^{33}$ We find that the $S_{\mathrm{d}}$ calculated from using $r=2.2$ also fits the measured $S$ remarkably well. Thus, the larger $S$ in mesoporous nanowires is influenced either by the strong energy dependence in carrier scattering $\left(\tau_{\mathrm{e}} \propto E^{1.7}\right)$ or carrier depletion and is unrelated to phonon drag.

The quenched phonon drag in solid NWs further allows us to directly infer the contribution of drag in the bulk from the difference between the data for the bulk and the nanowires. We find that phonon drag enhancement in the bulk is comparable to $k_{\mathrm{b}} / e$ (where $k_{\mathrm{b}}$ is the Boltzmann constant and $e$ is the electronic charge) at $300 \mathrm{~K}$ even at doping of $3 \times 10^{19} \mathrm{~cm}^{-3}$. This contradicts the narrative $\mathrm{e}^{34}$ that drag is relevant only at low temperature. Figure 4 plots this contribution across several doping levels. At low doping $\left(N<3 \times 10^{18} \mathrm{~cm}^{-3}\right)$, the temperature dependence is $S_{\mathrm{ph}} \sim T^{-2.1}(100-300 \mathrm{~K})$ as shown in Figure $4 \mathrm{a}$ and decreases at higher doping. The exponent agrees closely with the data of Geballe et al. ${ }^{7}$ and deviates from Herring's prediction ${ }^{6}$ of $\sim T^{-3.5}$ dependence. The discrepancy has previously been attributed to the finite size of samples but never satisfactorily explained. We now show that incorporating the appropriate MFP spectrum of phonons, $\Lambda(\omega)$, in the theory yields the exact observed temperature dependence.

To revise the formula for $S_{\mathrm{ph}}$, we require the relation for the electron energy flux resulting from the interacting with diffusing phonons of relevant frequencies. For this, we need to obtain the change in electron $\left(f=f_{0}+f_{0}{ }^{\prime} g_{\mathrm{e}} \cdot k_{x}\right)$ and phonon distributions $\left(N=N_{0}-N_{0}{ }^{\prime} g_{\mathrm{p}} \cdot q_{x}\right)$ in response to the temperature gradient $\mathrm{d} T / \mathrm{d} x$ and the resulting electric field $F_{x}{ }^{*}$. Here, $f_{0}(k)$ and $N_{0}(q)$ are distribution of electrons and phonons respectively at equilibrium while $g_{\mathrm{e}}$ and $g_{\mathrm{p}}$ are their respective deviations from equilibrium. $f_{0}{ }^{\prime}$ and $N_{0}{ }^{\prime}$ are the derivatives with respect to electron and phonon energy, respectively. $k_{x}$ and $q_{x}$ are electron and phonon wavevector, respectively, along the direction of transport. Using these distribution functions, we can write the Boltzmann transport equation for electrons ${ }^{16,35}$ under external forces and scattering processes including e-ph interaction as

Table 1. Summary of the Samples Used in the Seebeck Measurements with Their Room Temperature Thermal Conductivity with the Labels Px (p-type), Nx (n-type) and MS (mesoporous) SiNW arrays ${ }^{a}$

\begin{tabular}{ll} 
sample \# & \multicolumn{1}{c}{ est. doping } \\
BK1 & $3 \times 10^{19} \mathrm{~cm}^{-3} \mathrm{~B}$ \\
BK2 & $3 \times 10^{18} \mathrm{~cm}^{-3} \mathrm{~B}$ \\
BK3 & $6 \times 10^{19} \mathrm{~cm}^{-3} \mathrm{P}$ \\
BK4 & $2 \times 10^{17} \mathrm{~cm}^{-3} \mathrm{~B}$ \\
P1 & $3 \times 10^{18} \mathrm{~cm}^{-3} \mathrm{~B}$ \\
P2 & $8 \times 10^{18} \mathrm{~cm}^{-3} \mathrm{~B}$ \\
P3 & $5 \times 10^{19} \mathrm{~cm}^{-3} \mathrm{~B}$ \\
P4 & $1.5 \times 10^{19} \mathrm{~cm}^{-3} \mathrm{~B}$ \\
P5 & $2 \times 10^{19} \mathrm{~cm}^{-3} \mathrm{~B}$ \\
MS1 & $3 \times 10^{19} \mathrm{~cm}^{-3} \mathrm{~B}$ \\
N1 & $7 \times 10^{19} \mathrm{~cm}^{-3} \mathrm{P}$
\end{tabular}

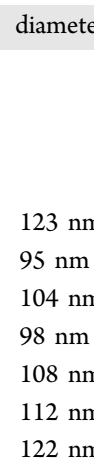

$123 \mathrm{~nm}$
$95 \mathrm{~nm}$
$104 \mathrm{~nm}$
$98 \mathrm{~nm}$
$108 \mathrm{~nm}$
$112 \mathrm{~nm}$
$122 \mathrm{~nm}$ length areal coverage

$1.3 \mu \mathrm{m}$
$1.25 \mu \mathrm{m}$
$2.3 \mu \mathrm{m}$
$1.2 \mu \mathrm{m}$
$2.1 \mu \mathrm{m}$
$1.6 \mu \mathrm{m}$
$1.4 \mu \mathrm{m}$
$20.3 \%$

$19.6 \%$

$18.6 \%$

$24.8 \%$

$28.6 \%$

$27.3 \%$

$17.2 \%$ $k(300 \mathrm{~K})$

$83 \mathrm{Wm}^{-1} \mathrm{~K}^{-1}$

$128 \mathrm{Wm}^{-1} \mathrm{~K}^{-1}$

$72 \mathrm{Wm}^{-1} \mathrm{~K}^{-1}$

$141 \mathrm{Wm}^{-1} \mathrm{~K}^{-1}$

$21 \mathrm{Wm}^{-1} \mathrm{~K}^{-1}$

$26 \mathrm{Wm}^{-1} \mathrm{~K}^{-1}$

$17 \mathrm{Wm}^{-1} \mathrm{~K}^{-1}$

$23 \mathrm{Wm}^{-1} \mathrm{~K}^{-1}$

$34 \mathrm{Wm}^{-1} \mathrm{~K}^{-1}$

$2.6 \mathrm{Wm}^{-1} \mathrm{~K}^{-1}$

$18 \mathrm{Wm}^{-1} \mathrm{~K}^{-1}$

${ }^{a} \mathrm{BK}$ are the bulk Si wafers of $550 \mu \mathrm{m}$ thick. 


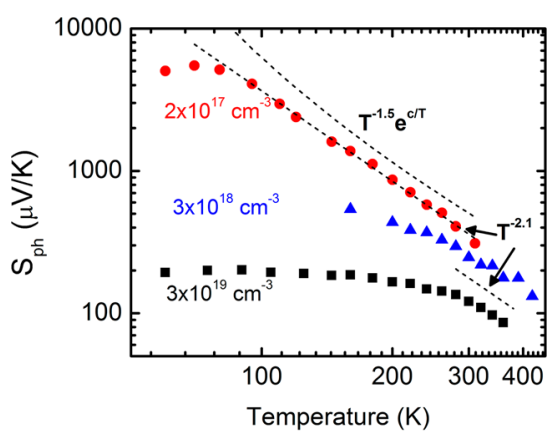

(a)

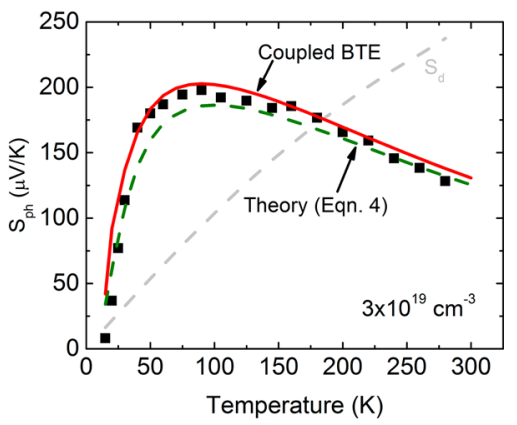

(b)

Figure 4. (a) The phonon drag component in bulk Si extracted at various doping concentrations with temperature dependent fits (dashed lines). (b) Equation 4 for $S_{\text {ph }}$ (dashed line) that accounts for phonon MFP spectrum agrees with the data for degenerate $S \mathrm{i}$. $S_{\mathrm{ph}}$ (solid line)from first order iterative solution of coupled BTE of electrons and phonons.

$$
\frac{f_{0}^{\prime} \hbar k_{x}}{m^{*}}\left(e F_{x}^{*}+\frac{E-E_{\mathrm{F}}}{T} \frac{\mathrm{d} T}{\mathrm{~d} x}\right)=-\frac{f_{0}^{\prime} g_{\mathrm{e}}}{\tau_{\mathrm{e}}}+\frac{f_{0}^{\prime} \hbar k_{x}}{4 m^{*} l_{\mathrm{ae}}}\left(\frac{1}{k^{3}} \int_{0}^{2 k} g_{\mathrm{p}}(q) q^{3} \mathrm{~d} q\right)
$$

Solving eq 3 along with BTE for phonons and imposing zero current condition $\int_{0}^{\infty} D(E) f_{0}^{\prime} g_{\mathrm{e}}(E) \mathrm{d} E=0$, we obtain the Seebeck coefficient $S=F_{x}{ }^{*} /(\mathrm{d} T / \mathrm{d} x)$. Here $l_{\mathrm{ae}}=\pi \hbar^{4} \rho u_{0}^{2} /$ $m^{* 2} \Xi^{2} k_{\mathrm{b}} T$ is the characteristic length of electron acoustic phonon scattering, where $\Xi$ is the deformation potential, $\rho$ is the crystal density, $m^{*}$ is the conductivity effective mass, and $u_{0}$ is phonon speed. When $g_{\mathrm{p}}$ is set to zero in eq 3 , the resulting $S$ is simply the diffusion component $S_{\mathrm{d}}$ shown in eq 2 . However, in the presence of a temperature gradient $\mathrm{d} T / \mathrm{d} x$ the nonzero $g_{\mathrm{p}}$ adds to the electron momentum (second term in RHS of eq 3 ) leading to phonon drag. The integral indicates that an electron in state $(\mathrm{E}, k)$ receives momentum from phonons of wavevector $q \leq 2 k$. To calculate $g_{\mathrm{p}}$, let us first assume that phonons are restored to the stationary state rapidly by phonon-phonon processes. In this case, $g_{\mathrm{p}}$ is independent of electron distribution $f$ and can be simply expressed as $g_{\mathrm{p}}(q)=\hbar u_{0}\left(\Lambda_{\mathrm{ph}} / T\right)(\mathrm{d} T / \mathrm{dx})$. Here, $\Lambda_{\text {ph }}$ is the phonon MFP calculated using Mattheissen's sum of all phonon scattering processes. We assume a linear dispersion, $\omega=u_{0} q$ for long wavelength phonons. $S_{\mathrm{ph}}$ is then obtained from the contribution of the integral containing $g_{p}$ in eq 3. Using the specific heat per frequency $C(\omega)=\hbar \omega\left(\mathrm{dN}_{0} /\right.$ $\mathrm{d} T)\left(\omega^{2} / u_{0}^{3}\right)$ and phonon-electron MFP $\Lambda_{\text {ep }}^{-1}=x f_{0}(q / 2) / 2 l_{\text {ae }}$ where $x=\hbar \omega / k_{\mathrm{b}} T$, the resulting phonon drag is

$$
\begin{aligned}
e T S_{\mathrm{ph}} & =\frac{\int_{0}^{\infty} \mathrm{d} E \tau_{\mathrm{e}} v_{x}^{2} D(E) f_{0}^{\prime} \frac{1}{2 k^{3}} \int_{0}^{2 k u_{0}} \frac{C(\omega) d \omega}{f_{0}\left(\frac{\omega}{2 u_{0}}\right)} \frac{\Lambda_{\mathrm{ep}}^{-1}}{\Lambda_{\mathrm{ph}}^{-1}}}{\int_{0}^{\infty} d E \tau_{e} v_{x}^{2} D(E) f_{0}^{\prime}} \\
& =\left\langle\frac{1}{2 k^{3}} \int_{0}^{2 k u_{0}} \frac{C(\omega) \mathrm{d} \omega}{f_{0}\left(\frac{\omega}{2 u_{0}}\right)} T \frac{\Lambda_{\mathrm{ep}}^{-1}}{\Lambda_{\mathrm{ph}}^{-1}}\right\rangle
\end{aligned}
$$

At low doping, momentum conservation dictates phonons only up to $\omega_{\mathrm{c}} \sim 1 \mathrm{THz}$ contribute to drag. ${ }^{36}$ At these frequencies, the phonon-phonon processes $\left(\Lambda_{\mathrm{pp}}^{-1} \sim \omega^{2} \mathrm{Te}^{-\mathrm{c} / \mathrm{T}}\right)$ dominate the phonon scattering rate $\Lambda_{\mathrm{ph}}^{-1}$ at sufficiently high temperatures. Using $\Lambda_{\mathrm{pp}}^{-1} \gg \Lambda_{\mathrm{ep}}^{-1}$ in eq 4 , we obtain $S_{\mathrm{ph}} \propto T^{-3 / 2} e^{\mathrm{c} / \mathrm{T}}$ with a weak dependence on carrier density. Figure 4a shows that this temperature trend correctly predicts the $\sim T^{-2.1}$ trend of the data for low-doped Si (Figure 4a). The data for $3 \times 10^{18} \mathrm{~cm}^{-3}$ also approaches a similar temperature trend for $T>200 \mathrm{~K}$ where Umklapp processes becomes dominates phonon scattering. Ignoring the MFP spectrum and using a single MFP $\bar{\Lambda}$ at $300 \mathrm{~K}^{10,37}$ under predicts the measure of $S_{\mathrm{ph}}$. However, revising the theory to include the MFP spectrum and averaging over frequencies up to the critical cutoff frequency $\omega_{c}$ is crucial in understanding the trend and measure of $S_{\mathrm{ph}}$.

At degenerate doping, the higher rate of electron-phonon scattering decreases the magnitude of $S_{\mathrm{ph}}$. Figure $4 \mathrm{~b}$ plots the data and the predictions from eq 4 to show good agreement with some deviations in the intermediate temperatures. Here, the deformation potential of longitudinal phonons, $\Xi=7 \mathrm{eV}$ is the only fitting parameter. High carrier densities $(n)$ broaden the phonon spectrum that interacts with electrons because the phonon critical frequency $\omega_{\mathrm{c}}\left(=2 u_{0} K_{\mathrm{F}}\right.$, where $K_{\mathrm{F}}$ is Fermi wavevector) scales as $n^{1 / 3}$. At $n \sim 3 \times 10^{19} \mathrm{~cm}^{-3}, \omega_{\mathrm{c}}$ increases to $\sim 2.5 \mathrm{THz}$. At low temperatures, the increased excitation of phonon modes below $\omega_{\mathrm{c}}\left(T_{\mathrm{c}}=\hbar \omega_{\mathrm{c}} / k_{\mathrm{b}}=125 \mathrm{~K}\right)$ competes against increased phonon scattering. Consistent with this picture, the $S_{\mathrm{ph}}$ data reaches a peak value of $\sim 2 k_{\mathrm{b}} / \mathrm{e}$ at $90 \mathrm{~K}$ as shown in Figure 4b. The temperature trend for $T>90 \mathrm{~K}$ is flatter in comparison to nondegenerate $\mathrm{Si}$ due to the dominance of phonon-electron scattering $\left(\Lambda_{\mathrm{ep}}^{-1} \sim \omega\right)$ over phonon-phonon scattering that has a steeper temperature dependence. At higher temperatures, $\Lambda_{\mathrm{pp}}^{-1}$ becomes comparable to $\Lambda_{\mathrm{ep}}^{-1}$ for $\omega<\omega_{\mathcal{\mathcal { O }}}$ and we observe a steeper decline in $S_{\mathrm{ph}}$ with a transition toward $\sim T^{-2.1}$ dependence. An extrapolation reveals that phonon drag does not vanish until $T \sim 600 \mathrm{~K}$.

Finally, we discuss the validity of the assumption in deriving eq 4. Strong electron-phonon scattering at degenerate doping sets up simultaneous nonequilibrium between electrons and phonons. ${ }^{17,36}$ The phonon imbalance $g_{p}$ now depends on $g_{e}$, requiring the solution of coupled Boltzmann equation of electrons and phonons. The Seebeck coefficient in this case cannot be decomposed into drag and diffusion. In metals, the coupled treatment is well established ${ }^{38}$ (where electrons at $E_{\mathrm{F}}$ only contribute to transport), but not straightforward for semiconductors. Using Conwell's iterative solution scheme ${ }^{35}$ for solving coupled $g_{\mathrm{e}}$ and $g_{\mathrm{p}}$ in degenerate semiconductors, we obtain the first order effect of this coupling on $S$ (see Supporting Information S6). Figure 4 b plots the resulting $S$ minus $S_{\mathrm{d}}$, showing a slight increase in phonon contribution to $S$ at $3 \times 10^{19} \mathrm{~cm}^{-3}$ peaking at $\sim 80 \mathrm{~K}$. This result shows that simultaneous nonequilibrium effects between electrons and phonons require much higher carrier concentration than $\sim 10^{19}$ 
$\mathrm{cm}^{-3}$ and that eq 4 is a good approximation for $S_{\mathrm{ph}}$ of $\sim 3 \times$ $10^{19} \mathrm{~cm}^{-3}$.

In conclusion, we have experimentally shown that phonon drag contributes significantly to $S$ in bulk $\mathrm{Si}$ even near room temperature and at degenerate doping. Boundary scattering of phonons in $\mathrm{Si}$ nanowires completely quenches drag and reduces $S$ by $\sim 34 \%$ and the power factor for optimal doping by at least $\sim 57 \%$ at $300 \mathrm{~K}$. We further expect the absence of $S_{\mathrm{ph}}$ will reduce Seebeck coefficient in nanoarchitectures like nanomeshes and thin films with characteristic length scales $\lesssim 100 \mathrm{~nm}$. In matching the magnitude and trend of $S_{\mathrm{ph}}$ from Herring's theory, we have revised the formulation to consider a phonon MFP spectrum. We further find that the issue of simultaneous nonequilibrium of electrons and phonons is not pronounced at $n \sim 10^{19} \mathrm{~cm}^{-3}$. Finally, phonon drag measurements can provide a means to further study the phonon MFP spectrum.

\section{ASSOCIATED CONTENT}

\section{S Supporting Information}

We include the details of fabrication of the nanowire array samples, secondary ion mass characterization, error analysis of Seebeck measurements, electrical measurements, calculation of diffusion component of Seebeck coefficient, and theory of phonon drag in bulk and low-dimensions. This material is available free of charge via the Internet at http://pubs.acs.org.

\section{AUTHOR INFORMATION}

\section{Corresponding Author}

*E-mail: sanjiv@illinois.edu.

\section{Notes}

The authors declare no competing financial interest.

\section{ACKNOWLEDGMENTS}

This work was supported by the Advanced Research Project Agency - Energy through Grant No. DOEDE- AR0000041PF-ARRA and the National Science Foundation through Grant No. NSF-CBET-09-54696-CAREER. We thank Dr. Timothy Spila for helping in characterization of the samples. We thank Dr. D. G. Cahill (UIUC) for helpful discussions.

\section{REFERENCES}

(1) Dresselhaus, M. S.; Chen, G.; Tang, M. Y.; et al. New directions for Low-Dimensional thermoelectric materials. Adv. Mater. 2007, 19 (8), 1043-1053.

(2) Heremans, J. P.; Dresselhaus, M. S.; Bell, L. E.; Morelli, D. T. When thermoelectrics reached the nanoscale. Nat. Nanotechnol. 2013, $8,471-473$.

(3) Vineis, C. J.; Shakouri, A.; Majumdar, A.; Kanatzidis, M. G. Nanostructured thermoelectrics: Big efficiency gains from small features. Adv. Mater. 2010, 22 (36), 3970-3980.

(4) Shakouri, A. Recent developments in semiconductor thermoelectric physics and materials. Ann. Rev. Mater. Res. 2011, 41, 399-431.

(5) Lyeo, H. K.; Khajetoorians, A. A.; Shi, L.; et al. Profiling the thermoelectric power of semiconductor junctions with nanometer resolution. Science 2004, 303 (5659), 816-818.

(6) Herring, C. Theory of the thermoelectric power of semiconductors. Phys. Rev. 1954, 96 (5), 1163.

(7) Geballe, T.; Hull, G. Seebeck effect in silicon. Phys. Rev. 1955, 98 (4), 940.

(8) Dismukes, J.; Ekstrom, L.; Steigmeier, E.; Kudman, I.; Beers, D. Thermal and electrical properties of heavily doped Ge-Si alloys up to $1300^{\circ}$ K. J. Appl. Phys. 1964, 35 (10), 2899-2907.
(9) Brinson, M.; Dunstant, W. Thermal conductivity and thermoelectric power of heavily doped n-type silicon. J. Phys. C: Solid State Physics. 1970, 3 (3), 483.

(10) Weber, L.; Gmelin, E. Transport properties of silicon. Appl. Phys. A 1991, 53 (2), 136-140.

(11) Heikes, R. R., Ure, R. W. Thermoelectricity: Science and Engineering; Interscience Publishers: New York, 1961; p 396.

(12) MacDonald DKC. Thermoelectricity: An Introduction to the Principles. Courier Dover Publications: New York, 2006.

(13) Conwell, E.; Weisskopf, V. Theory of impurity scattering in semiconductors. Phys. Rev. 1950, 77 (3), 388.

(14) Minnich, A. J.; Johnson, J.; Schmidt, A.; et al. Thermal conductivity spectroscopy technique to measure phonon mean free paths. Phys. Rev. Lett. 2011, 107 (9), 095901.

(15) Ward, A.; Broido, D. Intrinsic phonon relaxation times from first-principles studies of the thermal conductivities of si and ge. Phys. Rev. B 2010, 81 (8), 085205.

(16) Behnen, E. Quantitative examination of the thermoelectric power of n-type si in the phonon drag regime. J. Appl. Phys. 1990, 67 (1), 287-292.

(17) Sondheimer, E. The kelvin relations in thermo-electricity. Proc. R. Soc. London, Ser. A 1956, 234 (1198), 391-398.

(18) Yang, B.; Liu, J.; Wang, K.; Chen, G. Simultaneous measurements of seebeck coefficient and thermal conductivity across superlattice. Appl. Phys. Lett. 2002, 80 (10), 1758-1760.

(19) Curtin, B. M.; Fang, E. W.; Bowers, J. E. Highly ordered vertical silicon nanowire array composite thin films for thermoelectric devices. J. Electron. Mater. 2012, 1-8.

(20) Hochbaum, A. I.; Chen, R.; Delgado, R. D.; et al. Enhanced thermoelectric performance of rough silicon nanowires. Nature 2008, 451 (7175), 163-167.

(21) Boukai, A. I.; Bunimovich, Y.; Tahir-Kheli, J.; Yu, J.; Goddard Iii, W. A.; Heath, J. R. Silicon nanowires as efficient thermoelectric materials. Nature 2008, 451 (7175), 168-171.

(22) Singh, R; Bian, Z.; Shakouri, A.; et al. Direct measurement of thin-film thermoelectric figure of merit. Appl. Phys. Lett. 2009, 94 (21), 212508-212508-3.

(23) Bian, Z.; Zhang, Y.; Schmidt, H.; Shakouri, A. Thin film ZT characterization using transient harman technique. Int. Conf. Thermoelectr. 2005, 76-78.

(24) Björk, M. T.; Schmid, H.; Knoch, J.; Riel, H.; Riess, W. Donor deactivation in silicon nanostructures. Nat. Nanotechnol. 2009, 4 (2), 103-107.

(25) Azeredo, B.; Sadhu, J.; Ma, J.; et al. Silicon nanowires with controlled sidewall profile and roughness fabricated by thin-film dewetting and metal-assisted chemical etching. Nanotechnology 2013, 24 (22), 225305.

(26) Sadhu, J. S.; Tian, H.; Spila, T.; et al. Controllable doping and wrap-around contacts to electrolessly etched silicon nanowire arrays. Nanotechnology 2014, 25 (37), 375701.

(27) Balasundaram, K.; Sadhu, J. S.; Shin, J. C.; et al. Porosity control in metal-assisted chemical etching of degenerately doped silicon nanowires. Nanotechnology 2012, 23 (30), 305304.

(28) Pichanusakorn, P.; Bandaru, P. Nanostructured thermoelectrics. Mater. Sci. Eng., R 2010, 67 (2), 19-63.

(29) Fischetti, M. Effect of the electron-plasmon interaction on the electron mobility in silicon. Phys. Rev. B 1991, 44 (11), 5527.

(30) Lundstrom, M. Fundamentals of Carrier Transport; Cambridge University Press: New York, 2009.

(31) Ma, J.; Sinha, S. Thermoelectric properties of highly doped ntype polysilicon inverse opals. J. Appl. Phys. 2012, 112 (7), 07371907371999.

(32) Chattopadhyay, D.; Queisser, H. Electron scattering by ionized impurities in semiconductors. Rev. Mod. Phys. 1981, 53 (4), 745.

(33) Rao, P.; Schiff, E. A.; Tsybeskov, L.; Fauchet, P. Photocarrier drift-mobility measurements and electron localization in nanoporous silicon. Chem. Phys. 2002, 284 (1), 129-138. 
(34) Ryu, H. J.; Aksamija, Z.; Paskiewicz, D.; et al. Quantitative determination of contributions to the thermoelectric power factor in si nanostructures. Phys. Rev. Lett. 2010, 105 (25), 256601.

(35) Conwell, E. High Field Transport in Semiconductors; Academic Press: New York, 1967.

(36) Klemens, P. Theory of phonon drag thermopower. Int. Conf. Thermoelectr. 1996, 206-208.

(37) Krali, E.; Durrani, Z. A. Seebeck coefficient in silicon nanowire arrays. Appl. Phys. Lett. 2013, 102 (14), 143102.

(38) Bailyn, M. Phonon-drag part of the thermoelectric power in metals. Phys. Rev. 1967, 157 (3), 480.

(39) Maznev, A. Onset of size effect in lattice thermal conductivity of thin films. J. Appl. Phys. 2013, 113 (11), 113511. 